\title{
Spaces of continuous step functions over LOTS
}

\author{
by \\ Raushan Z. Buzyakova (Greensboro, NC)
}

\begin{abstract}
We investigate spaces $C_{\mathrm{p}}(\cdot, n)$ over LOTS (linearly ordered topological spaces). We find natural necessary conditions for linear Lindelöfness of $C_{\mathrm{p}}(\cdot, n)$ over LOTS. We also characterize countably compact LOTS whose $C_{\mathrm{p}}(\cdot, n)$ is linearly Lindelöf for each $n$. Both the necessary conditions and the characterization are given in terms of the topology of the Dedekind completion of a LOTS.
\end{abstract}

1. Introduction. We will study relations between covering properties of $C_{\mathrm{p}}(\cdot, n)$ over linearly ordered topological spaces (abbreviated as LOTS) and their Dedekind completions. Let us make one simple observation that will motivate the results of this paper. Let $L=(0,1] \cup[0,1)^{\prime}$ be the two arrows space of Aleksandrov. It is well known that $C_{\mathrm{p}}(L, 2)$ is not Lindelöf. This failure is witnessed by the following classical collection of functions: $F=\left\{f_{r}: r \in(0,1)\right\}$, where $f_{r}$ is 0 up to $r$ and 1 starting from $r^{\prime}$. If $g$ is a complete accumulation point for $F$ in $2^{L}$ then there exists $p^{\prime} \in[0,1)^{\prime}$ such that $g\left(\left[0^{\prime}, p^{\prime}\right]\right)=\{0\}$ and $g\left(\left(p^{\prime}, 1\right]\right)=\{1\}$ or $g\left(\left[0^{\prime}, p\right)\right)=\{0\}$ and $g([p, 1])=\{1\}$. That is, $g$ is discontinuous, and consequently, $F$ has no complete accumulation points in $C_{\mathrm{p}}(L, 2)$. However, if we consider the LOTS $L^{*}=L \backslash\left\{p, p^{\prime}\right\}$, and the family $F^{*}$ of the functions of $F$ restricted to $L^{*}$, then the new family has a complete accumulation point in $C_{\mathrm{p}}\left(L^{*}, 2\right)$, namely $\left.g\right|_{L^{*}}$. This is because the gap $\left\{p, p^{\prime}\right\}$ is a hole now, that is, not in $L^{*}$.

Roughly speaking, for Lindelöfness of $C_{\mathrm{p}}(\cdot, 2)$ it is necessary that "large collections of gaps accumulate to holes". It is of course also necessary that "large collections of holes accumulate to holes". We prove that in the class of countably compact LOTS, these two conditions together form a criterion for $C_{\mathrm{p}}(\cdot, n)$ to be linearly Lindelöf for each $n$ (Theorem 8 ). The second statement in topological language can be stated as follows: "If $C_{\mathrm{p}}(\cdot, 2)$ over

2000 Mathematics Subject Classification: 54C35, 54D20, 54F05, 54F65.

Key words and phrases: $C_{\mathrm{p}}(X, Y)$, linearly ordered topological space, Dedekind completion, linearly Lindelöf. 
a LOTS $L$ is linearly Lindelöf then so is the Dedekind remainder of $L$ ". To state both conditions in one topological sentence is not hard either (see Theorem 3) but requires one additional definition to be given later. In this paper we refine these two statements into sufficient/necessary conditions for linear Lindelöfness of $C_{\mathrm{p}}(\cdot, n)$ (or certain nice subspaces of it) over LOTS. The main results of this paper are given by Theorems 3,7 , and 8 , and Corollary 4 . We demonstrate how the conditions obtained can be used.

In notation and terminology we will follow [ENG] and [ARH]. As usual, $C_{\mathrm{p}}(X, Y)$ is the space of all continuous functions from $X$ to $Y$ endowed with the topology of pointwise convergence. All spaces considered are Tikhonov. A space $X$ is called linearly Lindelöf if every uncountable subset $A \subset X$ of regular cardinality has a complete accumulation point in $X$. Recall that a point $x \in X$ is a complete accumulation point (abbreviated as cap) for $A$ if every open neighborhood of $x$ meets $A$ in a subset of cardinality $|A|$. Special constructions and definitions about LOTS will be given at the beginning of the next section.

2. Results. Given a LOTS $L$, an ordered pair $\langle A, B\rangle$ of disjoint subsets of $L$ is called a Dedekind section if $A \cup B=L, \sup A$ does not exist, $\inf B$ does not exist, and $A$ is to the left of $B$. A pair $\langle L, \emptyset\rangle$ (resp. $\langle\emptyset, L\rangle)$ is also a Dedekind section if $\sup L(\operatorname{resp} . \inf L)$ does not exist. The Dedekind completion of $L$ is denoted by $c L$, and the set $c L \backslash L$ of all Dedekind sections by $d L$.

Let us quickly recall the definition of Dedekind completion. The set $c L$ is the union of $L$ and the set $d L$ of all Dedekind sections of $L$. The order on $c L$ is very natural. The order on elements of $L$ is not changed. If $x \in L$ and $y=\langle A, B\rangle \in d L$ then $x$ is less (resp. greater) than $y$ if $x \in A$ (resp. $x \in B$ ). If $x=\left\langle A_{1}, B_{1}\right\rangle$ and $y=\left\langle A_{2}, B_{2}\right\rangle$ are elements of $d L$, then $x$ is less than $y$ if $A_{1}$ is a proper subset of $A_{2}$.

An ordered pair $\langle a, b\rangle$ of elements of $L$ is called a gap if $a<b$ and there is no other element between $a$ and $b$. The least and greatest elements in $c L$ will be denoted by $-\infty$ and $\infty$, respectively. When we work with a particular LOTS $L$, by $(a, b)$ we will mean the interval in the Dedekind completion $c L$. The trace of this interval on a subspace $X$ of $c L$ (on $L$ in particular) will be denoted by $(a, b)_{X}$. This agreement is rather unusual but is convenient for our discussion. A function $f$ is a step function (with finitely many steps) from a LOTS $L$ to $X$ if there exist points $a_{1}, \ldots, a_{m} \in c L$ (called transition points) and elements $i_{0}, \ldots, i_{m} \in X$ such that $f\left(\left[-\infty, a_{1}\right]_{L}\right)=\left\{i_{0}\right\}, f\left(\left(a_{1}, a_{2}\right]_{L}\right)=\left\{i_{1}\right\}, \ldots, f\left(\left(a_{m}, \infty\right]_{L}\right)=\left\{i_{m}\right\}$. Regarding transition points, we always assume that none of the intervals $\left[-\infty, a_{1}\right]_{L},\left(a_{1}, a_{2}\right]_{L}, \ldots,\left(a_{m}, \infty\right]_{L}$ is empty and $i_{k} \neq i_{k+1}$. It is clear that if $f$ is a continuous step function from $L$ to a Hausdorff space, then every 
transition point of $f$ is either in the Dedekind remainder $d L$ or is the left endpoint of a gap in $L$.

Now let us define the key structure for this paper. Given a LOTS $L$, we denote by $T(L)=(T, \mathcal{T})$ the space obtained as follows. The underlying set is the subset $T$ of $c L$ that consists of all Dedekind sections as well as left endpoints of gaps of $L$. That is, $T=d L \cup\{$ left endpoints of gaps in $L\}$. The topology $\mathcal{T}$ of $T(L)$ is defined using the Bing-Hanner construction (see [ENG, 5.1.22]). Namely, base neighborhoods at points of $d L$ are those from the subspace topology on $T$, while all other points are declared isolated. Let us describe another way to obtain $T(L)$. First insert a new single point in each gap and denote the resulting LOTS by $L^{\prime}$. The subspace of $c L^{\prime}$ that consists of all Dedekind sections and the inserted points is clearly naturally homeomorphic to $T(L)$. Observe that if $f$ is a step function with finitely many steps from $L$ to $\{0, \ldots, n-1\}$ then $f$ is continuous iff all transition points of $f$ are in $T$.

To get used to $T(L)$, let us see what it looks like for some simple spaces. It is clear that for compact LOTS $L, d L$ is empty. Therefore, $T(L)$ is simply a discrete space that has the same cardinality as the set of all gaps in $L$. As another example, let $L=(0,1] \cup[0,1)^{\prime}$ be the two arrows of Aleksandrov and let $A \subset(0,1)$ be any subset. Let $L_{A}=L \backslash\left\{r, r^{\prime}: r \in A\right\}$. The space $T\left(L_{A}\right)$ can be obtained from $(0,1)$ by retaining Euclidean neighborhoods at points of $A$ and declaring points of $(0,1) \backslash A$ isolated. This is a classical Michael-type space and is well investigated.

Let us start our study with the following technical fact.

LEMMA 1. Let $L$ be a LOTS and let $F \subset C_{\mathrm{p}}(L, n)$ be a family of step functions with at most $m$ transition points each. If a non-constant $f \in$ $C_{\mathrm{p}}(L, n)$ is limit for $F$, then $f$ is a step function with at most $m$ transition points. Moreover, if for any distinct $g, h \in F$, the sets of transition points of $g$ and $h$ are disjoint then all transition points of $f$ are in $d L$.

Proof. First, there is no strictly increasing sequence $p_{1}<\cdots<p_{m+2}$ in $L$ such that $f\left(p_{i}\right) \neq f\left(p_{i+1}\right)$ for $i=1, \ldots, m+1$. Indeed, the existence of such a sequence implies that the neighborhood $U_{f}=\left\{g: g\left(p_{i}\right)=\right.$ $\left.f\left(p_{i}\right), i=1, \ldots, m+2\right\}$ does not contain step functions with fewer than $m+1$ transition points, a contradiction with $f$ being a limit point for $F$. The rest of the first part is now clear. Simply take the suprema (in $c L$ ) of the maximal intervals (except the last one) on which the function is constant.

For the "moreover" part assume the contrary and let $a \in L$ be a transition point of $f$. Then there exists $b$ such that $\langle a, b\rangle$ is a gap in $L$. Since $a$ is a transition point, $f(a) \neq f(b)$. The set $U_{f}=\{g: g(a)=f(a), g(b)=f(b)\}$ is a neighborhood of $f$. If $g \in U_{f}$ is a step function then $a$ is a transition point 
of $g$ as well. Since $U_{f}$ contains infinitely many elements of $F$, they must all share a transition point, contradicting the hypothesis.

The next theorem is the base induction step for the proof of our main necessary condition (Theorem 3).

Theorem 2. Let $L$ be a LOTS. If $C_{\mathrm{p}}(L, 2)$ is linearly Lindelöf then so is $T(L)$.

Proof. Fix any $A \subset T$ of uncountable regular cardinality. For each $a \in A$, the function $f_{a}$ defined to be 0 on $[-\infty, a]_{L}$ and 1 on $(a, \infty]_{L}$ is either constant or a continuous step function because the transition point is in $T$. Clearly, for different $a$ 's, the $f_{a}$ 's differ. Therefore, $F_{A}=\left\{f_{a}: a \in A\right\}$ has cardinality $|A|$. Since $C_{\mathrm{p}}(L, 2)$ is linearly Lindelöf, there exists $f \in C_{\mathrm{p}}(L, 2)$ that is a cap for $F_{A}$. By Lemma $1, f$ is either constant or a step function with one transition point.

Let $f \equiv 0$. Let us show that $\infty$ is a cap for $A$ in $T(L)$. First, $\infty \in d L$. Indeed, if it were in $L$ then the set $\{g: f(\infty)=0\}$ would be a neighborhood of $f$. But this neighborhood does not contain a single element of $F_{A} \backslash\{f\}$, since each function in $F_{A} \backslash\{f\}$ is eventually 1 . Since $\infty \in d L$, its base neighborhoods in $T(L)$ are from the subspace topology on $T$. So, fix any $x \in L$ below $\infty$. We need to show that $(x, \infty]$ contains $|A|$ elements of $A$. The set $U_{f}=\{g: g(x)=0\}$ is a neighborhood of $f$, hence meets $F_{A}$ in a subset of cardinality $|A|$. If $f_{a} \in U_{f} \cap F_{A}$, then the transition point $a$ is in $(x, \infty]$. Similarly, $-\infty$ is a cap for $A$ in $T(L)$ if $f \equiv 1$.

If $f$ is not constant, then there exists $c \in T$ such that $f\left([-\infty, c]_{L}\right)=\{0\}$ and $f\left((c, \infty]_{L}\right)=\{1\}$. By the "moreover" part of Lemma 1, $c \in d L$. Also, since $f$ is not constant, $c \neq \pm \infty$. Therefore, base neighborhoods of $c$ in $T(L)$ are of the form $(x, y)_{T}$, where $c \in(x, y)$. Take any interval $(x, y) \ni c$ with end-points in $L$. The set $U_{f}=\{g: g(x)=0, g(y)=1\}$ contains $f$, and therefore $|A|$ elements of $F_{A}$. If $f_{a} \in U_{f}$ then the transition point $a$ has to be in $(x, y)$. Therefore, $c$ is a cap for $A$ in $T(L)$.

Theorem 3. Let $L$ be a LOTS. If $C_{\mathrm{p}}(L, n+1)$ is linearly Lindelöf then so is $(T(L))^{n}$.

Proof. Theorem 2 proves the statement for $n=1$. Suppose that for each natural number $k$ less than $n>1$, the statement is true. Fix an uncountable regular cardinal number $\tau$ and $A \subset T^{n}$ of cardinality $\tau$. Each $a \in A$ has coordinate representation $\left\langle a_{1}, \ldots, a_{n}\right\rangle$. There is $A^{\prime} \subset A$ of size $\tau$ such that for any $a, b \in A^{\prime}$, the coordinate-wise correspondence $a_{1} \leftrightarrow b_{1}, a_{2} \leftrightarrow b_{2}, \ldots$ is order-preserving (order on $a_{i}$ 's and $b_{i}$ 's is that from $c L$ ).

CASE I: For some element of $A^{\prime}$, at least two coordinates coincide. This means that the same coordinates coincide for any other element of $A^{\prime}$. We 
may assume that for each $a \in A^{\prime}, a_{1}=a_{2}$. The set $S=\left\{\left\langle a_{2}, \ldots, a_{n}\right\rangle\right.$ : $\left.\left\langle a_{1}, a_{2}, \ldots, a_{n}\right\rangle \in A^{\prime}\right\}$ is a subset of $T^{n-1}$. The set $S$ has cardinality $\tau$, because if the first coordinates of $a$ and $b$ in $A^{\prime}$ differ, their second coordinates differ too. Since $C_{\mathrm{p}}(L, n)$ is a closed subspace of $C_{\mathrm{p}}(L, n+1), C_{\mathrm{p}}(L, n)$ is also linearly Lindelöf. Therefore, by inductive assumption, $(T(L))^{n-1}$ is linearly Lindelöf. Thus, there exists a cap $\left\langle c_{2}, \ldots, c_{n}\right\rangle$ for $S$ in $(T(L))^{n-1}$. Clearly, $\left\langle c_{2}, c_{2}, \ldots, c_{n}\right\rangle$ is in $T^{n}$ and is a cap for $A^{\prime}$ in $(T(L))^{n}$.

CASE II: There exists a $\tau$-sized subset $A^{*}$ of $A$ and $i^{*} \in\{1, \ldots, n\}$ such that the $i^{*}$ th coordinates of any $a, b \in A^{*}$ coincide and are equal to some fixed element $c_{1}$. We may assume that $i^{*}=1$. By induction assumption, there exists $\left\langle c_{2}, \ldots, c_{n}\right\rangle \in T^{n-1}$ which is a cap for

$$
\left\{\left\langle a_{2}, \ldots, a_{n}\right\rangle:\left\langle a_{1}, a_{2}, \ldots, a_{n}\right\rangle \in A^{*}\right\}
$$

in $(T(L))^{n-1}$. Clearly, $\left\langle c_{1}, \ldots, c_{n}\right\rangle$ is a cap for $A^{*}$ in $(T(L))^{n}$.

CASE III: Neither Case I nor Case II takes place. By the $\Delta$-system lemma there exists a set $S$ (a root) and a $\tau$-sized subset $A^{\prime \prime}$ of $A^{\prime}$ such that for any $\left\langle a_{1}, \ldots, a_{n}\right\rangle,\left\langle b_{1}, \ldots, b_{n}\right\rangle \in A^{\prime \prime}$ the coordinate sets $\left\{a_{1}, \ldots, a_{n}\right\}$ and $\left\{b_{1}, \ldots, b_{n}\right\}$ meet in $S$. If $S$ is not empty, then there exists $i^{*} \in\{1, \ldots, n\}$ and a $\tau$-sized subset $A^{*}$ of $A^{\prime \prime}$ such that the $i^{*}$ th coordinates of any two elements of $A^{*}$ coincide. But this is not possible in Case III. Therefore, $S$ is empty. Thus we may assume that for any distinct $a, b \in A^{\prime}$ no coordinate of $a$ is equal to any coordinate of $b$.

Since it is not Case I, all coordinates of some $a^{*} \in A^{\prime}$ are different. Renumber the coordinate axes of $T^{n}$ in such a manner that the coordinates of $a^{*}$ are strictly increasing. Since the coordinatewise correspondence is orderpreserving on elements of $A^{\prime}$, the coordinates of any point in $A^{\prime}$ are now strictly increasing. For each $a \in A^{\prime}$ define $f_{a}$ as follows:

$$
f_{a}\left(\left[-\infty, a_{1}\right]_{L}\right)=\{0\}, \quad f_{a}\left(\left(a_{1}, a_{2}\right]_{L}\right)=\{1\}, \ldots, f_{a}\left(\left(a_{n}, \infty\right]_{L}\right)=\{n\} .
$$

Since $a_{i}<a_{i+1}, f_{a}$ is defined on all of $L$. Since $a_{1}, \ldots, a_{n} \in T, f_{a}$ is continuous for each $a \in A^{\prime}$. Clearly, for distinct $a, b \in A^{\prime}$ the corresponding functions are distinct. Therefore, $F_{A^{\prime}}=\left\{f_{a}: a \in A^{\prime}\right\}$ has cardinality $\tau$. By hypothesis, there exists a cap $f$ for $\left\{f_{a}: a \in A^{\prime}\right\}$. By Lemma $1, f$ is either constant or a step function with at most $n$ transition points. As we remarked earlier, distinct $a$ and $b$ in $A^{\prime}$ have disjoint coordinate sets. Therefore, by Lemma 1, all transition points of $f$ (if there are any) are in $d L$.

Define the point $c=\left\langle c_{1}, \ldots, c_{n}\right\rangle$ as follows: $c_{i}=-\infty$ if $f(x) \geq i$ for all $x$; otherwise, $c_{i}=\sup _{c L}\{x \in L: f(x)<i\}$. We want to show that $c$ is a cap for $A^{\prime}$ in $(T(L))^{n}$.

First, let us show that $c \in(d L)^{n}$. If $c_{i}$ is neither $\infty$ nor $-\infty$, then $c_{i}$ is a transition point of $f$, and therefore is in $d L$. Suppose $c_{i}=\infty$ and let us show that $\infty \in d L$. Assume the contrary. By the definition of $c_{i}$, 
$f\left(c_{i}\right)=f(\infty)<i \leq n$. Since no two distinct elements of $A^{\prime}$ share a single coordinate, the $n$th coordinates of all elements of $A^{\prime}$, except maybe one, are below $\infty$. Therefore, $f_{a}(\infty)=n$ for all $a \in A^{\prime}$, contradicting $f$ being a cap for $F_{A^{\prime}}$. Suppose now $c_{i}=-\infty$. Assume $-\infty \in L$. Then $f(-\infty) \geq i \geq 1$. But $f_{a}(-\infty)=0$ for all $a$, a contradiction.

Now let us show that $c$ is a cap for $A^{\prime}$ in $(T(L))^{n}$. Since base neighborhoods at points of $d L$ in $T(L)$ are those from the subspace topology, it is enough to show that $c$ is a cap for $A^{\prime}$ in $(c L)^{n}$. Fix any closed box $\left[x_{1}, y_{1}\right] \times \cdots \times\left[x_{n}, y_{n}\right]$ containing $\left\langle c_{1}, \ldots, c_{n}\right\rangle$ in its interior and such that $x_{i} \in L$ if $c_{i} \neq-\infty$ and $y_{i} \in L$ if $c_{i} \neq \infty$. Let $U_{f} \subset C_{\mathrm{p}}(L, n+1)$ be the neighborhood of $f$ that contains all functions that coincide with $f$ at $x_{i}$ 's and $y_{i}$ 's distinct from $\pm \infty$. Since $U_{f}$ is an open neighborhood of $f$, it contains $\tau$ elements of $F_{A^{\prime}}$. Let us show that for any $f_{a} \in U_{f}, a$ is in the fixed box. We need to show that the $i$ th coordinate $a_{i}$ of $a$ is in $\left[x_{i}, y_{i}\right]$. If $c_{i}=\infty$ then $f\left(x_{i}\right)<i$. Since $f_{a} \in U_{f}, f_{a}\left(x_{i}\right)<i$. By the definition of $f_{a}$, if $f_{a}(x)<i$ then $x \leq a_{i}$. Therefore, $a_{i} \in\left[x_{i}, \infty\right]=\left[x_{i}, c_{i}\right]=\left[x_{i}, y_{i}\right]$. If $c_{i}=-\infty$, then $f\left(y_{i}\right) \geq i$. Since $f_{a} \in U_{f}, f_{a}\left(y_{i}\right) \geq i$, which means $y_{i} \in\left(a_{i}, \infty\right]$. Therefore, $a_{i} \in\left[-\infty, y_{i}\right]=\left[c_{i}, y_{i}\right]=\left[x_{i}, y_{i}\right]$. If $c_{i}$ is between infinities, then $f\left(x_{i}\right)<i$ and $f\left(y_{i}\right) \geq i$. Since $f_{a} \in U_{f}$, the same inequalities hold for $f_{a}$. Therefore, by the definition of $f_{a}, a_{i}$ must be in $\left[x_{i}, y_{i}\right]$.

One might have an impression that this necessary condition is rather artificial and is hard to use. Let us demonstrate the condition at work. Nahmanson [NAH] proved that $C_{\mathrm{p}}$ over a compact LOTS $L$ is Lindelöf iff $L$ is metrizable. Let us show how to derive a weaker version of Nahmanson's theorem from Theorem 2. As observed earlier, if $L$ is a compact LOTS, then $T(L)$ is discrete and has the same cardinality as the set of all gaps in $L$. Clearly, a discrete space is linear Lindelöf iff it is countable. Applying Theorem 2 we have the following statement: "Let $L$ be a zero-dimensional compact LOTS. Then $C_{\mathrm{p}}(L, 2)$ is linearly Lindelöf iff $L$ is metrizable". This application is certainly not very impressive but serves the demonstration purpose.

Since $d L$ is a closed subspace of $T(L)$ we have the following.

Corollary 4. Let L be a LOTS. If $C_{\mathrm{p}}(L, n+1)$ is linearly Lindelöf then $(d L)^{n}$ is linearly Lindelöf.

The conclusions in the above two theorems suggest the following question.

QUESTION 5. Let $L$ be a LOTS with $C_{\mathrm{p}}(L, n+1)$ Lindelöf (or linearly Lindelöf $)$. Is $(d L)^{n}$ Lindelöf? Is $(T(L))^{n}$ Lindelöf?

For $n=1$, the answer to this question is "yes". Indeed, since $L$ is a LOTS, $T$ is hereditarily countably paracompact [ENG, 5.5.22]. Since the 
Bing-Hanner construction does not destroy hereditary countable paracompactness, $T(L)$ is a countably paracompact linearly Lindelöf space. (It is proved in [ENG, 5.1.22] that the Bing-Hanner construction preserves hereditary paracompactness. For hereditary countable paracompactness, the proof is almost the same with obvious modifications.) Hence, by the result of Mishchenko [MIS], $T(L)$ is Lindelöf.

Corollary 6. Let $L$ be a LOTS. If $C_{\mathrm{p}}(L, n)$ is linearly Lindelöf for each $n$ then $(T(L))^{n}$ is linearly Lindelöf for each $n$.

It is natural to ask if the implication in the previous corollary can be reversed. No it cannot be. Let $S=\{0\} \cup\{1 / n: n \in \omega \backslash\{0\}\}$. Remove from the lexicographical product $\omega_{1} \times_{l} S$ all points of the form $\langle\alpha, 0\rangle$, where $\alpha$ is limit. The resulting space $L$ is a LOTS. Any neighborhood of the point $\infty$ in $T(L)$ contains all of $T(L)$ except maybe a countable part of it. Therefore, $T(L)$ is a continuous image of the Aleksandrov one-point Lindelöfication of an $\omega_{1}$-sized discrete space, and therefore, a Lindelöf P-space. Hence, $(T(L))^{n}$ is Lindelöf for each $n$. However, $C_{\mathrm{p}}(L, 2)$ is not linearly Lindelöf. Indeed, $L$ contains a clopen subspace homeomorphic to the free sum of $\omega_{1}$ copies of $S$. Therefore, $C_{\mathrm{p}}(L, 2)$ contains a closed subspace homeomorphic to $\left(C_{\mathrm{p}}(S, 2)\right)^{\omega_{1}}$. Since $C_{\mathrm{p}}(S, 2)$ is not compact, its uncountable power cannot be Lindelöf. Since every linearly Lindelöf space of weight $\omega_{1}$ is Lindelöf and the weight of $\left(C_{\mathrm{p}}(S, 2)\right)^{\omega_{1}}$ is $\omega_{1}$, the power cannot be linearly Lindelöf either.

In Theorem 8 below, we show that the converse of Corollary 6 does nevertheless hold for countably compact LOTS. In Theorem 7 , we show that linear Lindelöfness of $(T(L))^{n}$ for each $n$ implies linear Lindelöfness of certain very nice subspaces of $C_{\mathrm{p}}(L, n)$.

For the next theorem, we denote by $S_{\mathrm{p}}(L, n)$ the subspace of $C_{\mathrm{p}}(L, n)$ that consists of all step functions with finitely many steps and all constant functions. An open set $U \subset C_{\mathrm{p}}(L, n)$ will be called standard if there exist $x_{1}, \ldots, x_{m} \in L$ and $b_{1}, \ldots, b_{m} \in\{0, \ldots, n-1\}$ such that $U=\left\{g: g\left(x_{i}\right)=\right.$ $\left.b_{i}, i=1, \ldots, m\right\}$. If $x$ is one of $x_{i}$ 's we say that $U$ depends on $x$. In other words, $U$ depends on $x$ if $x$ participates in the definition of $U$.

TheOREM 7. Let L be a LOTS and let $(T(L))^{n}$ be linearly Lindelöf for each $n$. Then $S_{\mathrm{p}}(L, n)$ is linearly Lindelöf for each $n$.

Proof. Fix any $n>0$ and an uncountable regular cardinal $\tau$. Let $F \subset$ $S_{\mathrm{p}}(L, n)$ be of size $\tau$. There exist $F^{\prime} \subset F$ of size $\tau, m>0$, and $i_{0}, \ldots, i_{m} \in$ $\{0,1, \ldots, n-1\}$ such that each $f \in F^{\prime}$ is a function with $m$ transition points and

$$
f\left(\left[-\infty, a_{1}^{f}\right]_{L}\right)=\left\{i_{0}\right\}, \quad f\left(\left(a_{1}^{f}, a_{2}^{f}\right]_{L}\right)=\left\{i_{1}\right\}, \ldots, f\left(\left(a_{m}^{f}, \infty\right]_{L}\right)=\left\{i_{m}\right\},
$$

where $a_{1}^{f}, \ldots, a_{m}^{f}$ are the transition points of $f$. Since $f$ is continuous, all 
$a_{i}^{f}$ 's are in $T$. Since $i_{0}, \ldots, i_{m}$ are the same for all functions in $F^{\prime}$, different functions in $F^{\prime}$ must have different collections of transition points. Therefore, $A_{F^{\prime}}=\left\{\left\langle a_{1}^{f}, \ldots, a_{m}^{f}\right\rangle: f \in F^{\prime}\right\}$ has cardinality $\tau$. By hypothesis, there exists $c=\left\langle c_{1}, \ldots, c_{m}\right\rangle \in T^{m}$ which is a complete accumulation point for $A_{F^{\prime}}$ in $(T(L))^{m}$. It is clear that $c_{i} \leq c_{i+1}$. Therefore, the following function $h$ is defined on all of $L$ :

$$
h\left(\left[-\infty, c_{1}\right]_{L}\right)=\left\{i_{0}\right\}, \quad h\left(\left(c_{1}, c_{2}\right]_{L}\right)=\left\{i_{1}\right\}, \ldots, h\left(\left(c_{m}, \infty\right]_{L}\right)=\left\{i_{m}\right\} .
$$

This function is continuous since all $c_{i}$ 's are in $T$. Clearly, $h \in S_{\mathrm{p}}(L, n)$. Let us show that $h$ is a cap for $F^{\prime}$. Let $U_{h}$ be a standard neighborhood of $h$. We may assume that $U_{h}$ satisfies the following conditions (make it smaller if needed):

P1. If $c_{j}$ is in $L$ then $U_{h}$ depends on $c_{j}$.

P2. If $c_{j} \neq-\infty$ (resp. $\left.c_{j} \neq \infty\right)$ then there exists $z \in(-\infty, \infty)_{L}$ such that $U_{h}$ depends on $z$ and $z \leq c_{j}$ (resp. $c_{j} \leq z$ ). Let us show this for $c_{j} \neq-\infty$. If $c_{j} \in L$ put $z=c_{j}$. Otherwise, $c_{j} \in d L \backslash\{-\infty\}$. Therefore, $c_{j}$ is limit for $\left[-\infty, c_{j}\right)_{L}$, so pick any $z$ in this interval.

P3. If $c_{j}<c_{j+1}$ then there are $x, y \in L$ such that $U_{h}$ depends on $x, y$ and $c_{j} \leq y<x \leq c_{j+1}$.

Put $x_{j}=-\infty$ if $c_{j}=-\infty$ and $x_{j}=\max \left\{x \leq c_{j}: U_{h}\right.$ depends on $\left.x\right\}$ otherwise ( $\max$ is evaluated for a non-empty set due to P2). Put $y_{j}=\infty$ if $c_{j}=\infty$, and $y_{j}=\min \left\{y \geq c_{j}: U_{h}\right.$ depends on $\left.y\right\}$ otherwise. Due to $\mathrm{P} 1$, if $c_{j} \in L$ then $\left[x_{j}, y_{j}\right]=\left\{c_{j}\right\}$, which is an open neighborhood of $c_{j}$ in $T(L)$. If $c_{j} \in d L$ then $\left[x_{j}, y_{j}\right]$ has $c_{j}$ in its interior in $c L$. Therefore the interior of the box $B=\left[x_{1}, y_{1}\right]_{T} \times \cdots \times\left[x_{m}, y_{m}\right]_{T}$ in $(T(L))^{m}$ contains the point $c=\left\langle c_{1}, \ldots, c_{m}\right\rangle$. Since $c$ is a cap for $A_{F^{\prime}}, B$ meets $A_{F^{\prime}}$ in a subset of size $\tau$. Let us show that $f$ belongs to $U_{h}$ for $\tau$ many $a^{f} \in B \cap A_{F^{\prime}}$. Fix $a^{f}=\left\langle a_{1}^{f}, \ldots, a_{m}^{f}\right\rangle \in B \cap A_{F^{\prime}}$. Fix any $z$ such that $U_{h}$ depends on $z$. We need to show that $f(z)=h(z)$.

CASE I: $z=x_{j}$ for some $j$. Let $j^{\prime}$ be the smallest such that $c_{j}=c_{j^{\prime}}$. We have $c_{j^{\prime}} \in\left[x_{j^{\prime}}, y_{j^{\prime}}\right]=\left[x_{j}, y_{j}\right]$. Since $a^{f} \in B$, we have $a_{j^{\prime}}^{f} \in\left[x_{j^{\prime}}, y_{j^{\prime}}\right]$. If $j^{\prime}=1$ then $x_{j^{\prime}}$ belongs to $\left[-\infty, c_{1}\right]$ and to $\left[-\infty, a_{1}^{f}\right]$, and therefore, $f\left(x_{j^{\prime}}\right)=i_{0}=$ $h\left(x_{j^{\prime}}\right)$. If $j^{\prime}>1$ then, by the choice of $j^{\prime}, c_{j^{\prime}-1}<c_{j^{\prime}}$. By P3, $y_{j^{\prime}-1}<x_{j^{\prime}}$. Since $a^{f} \in B$, we have $a_{j^{\prime}-1}^{f} \in\left[x_{j^{\prime}-1}, y_{j^{\prime}-1}\right]$ and $a_{j^{\prime}}^{f} \in\left[x_{j^{\prime}}, y_{j^{\prime}}\right]$. Therefore, $x_{j^{\prime}} \in\left(a_{j^{\prime}-1}^{f}, a_{j^{\prime}}^{f}\right]$. Thus, $f\left(x_{j^{\prime}}\right)=i_{j^{\prime}-1}$. Also since $x_{j^{\prime}} \in\left(c_{j^{\prime}-1}, c_{j^{\prime}}\right]$, we have $h\left(x_{j^{\prime}}\right)=i_{j^{\prime}-1}$. Since $x_{j^{\prime}}=x_{j}$, we have $f\left(x_{j}\right)=h\left(x_{j}\right)$.

CASE II: $z=y_{j}$ for some $j$. If $y_{j}=c_{j}$ then $x_{j}=y_{j}$. Therefore $z=x_{j}$ and Case I applies. Suppose now $y_{j}>c_{j}$, in which case $c_{j}$ is in $d L$.

Let $B^{*}=\left[x_{1}^{*}, y_{1}^{*}\right]_{T} \times \cdots \times\left[x_{m}^{*}, y_{m}^{*}\right]_{T}$ be a sub-box of the box $B$ whose interior contains $c$ and that has the following property: 
P4. If $c_{i} \in d L$ and $c_{i}$ is limit in $c L$ from the right (resp. left) then $y_{i}^{*}<y_{i}$ (resp. $x_{i}<x_{i}^{*}$ ).

Since $B^{*}$ is a neighborhood of $c$, it contains $\tau$ elements of $A_{F^{\prime}}$. So we may assume that the fixed $a^{f}$ is in fact in $B^{*}$. Let $j^{\prime}$ be the largest such that $c_{j}=c_{j^{\prime}}$. Since $y_{j}>c_{j}$, we have $y_{j} \in\left(c_{j^{\prime}}, \infty\right]$. Thus, if $j^{\prime}=m$ then $h\left(y_{j}\right)=$ $i_{m}$. Now assume $j^{\prime}<m$. By P3, $y_{j^{\prime}} \leq c_{j^{\prime}+1}$. Therefore, $y_{j} \in\left(c_{j^{\prime}}, c_{j^{\prime}+1}\right]$ and $h\left(y_{j}\right)=i_{j^{\prime}}$. Let us prove that $f$ has the same values. Since $a^{f} \in B^{*}$, we have $a_{j^{\prime}}^{f} \in\left[x_{j^{\prime}}^{*}, y_{j^{\prime}}^{*}\right]$. Since $c_{j}<y_{j}$ and $c_{j^{\prime}}=c_{j}$, we conclude that $c_{j^{\prime}} \in d L$ and is below $\infty$. By P4, $a_{j^{\prime}}^{f} \leq y_{j^{\prime}}^{*}<y_{j^{\prime}}$. Therefore, $y_{j^{\prime}}$ is in $\left(a_{j^{\prime}}^{f}, \infty\right]$ and so is $y_{j}$. Thus, if $j^{\prime}=m$ then $f\left(y_{j}\right)=i_{m}$. If $j^{\prime}<m$ then $a_{j^{\prime}+1}^{f} \in\left[x_{j^{\prime}+1}, y_{j^{\prime}+1}\right]$ and $y_{j^{\prime}} \leq x_{j^{\prime}+1}$. Therefore, $y_{j} \in\left(a_{j^{\prime}}^{f}, a_{j^{\prime}+1}^{f}\right]$ and $f\left(y_{j}\right)=i_{j^{\prime}}$.

CASE III: Neither Case I nor Case II takes place. Then $z$ is either in $\left(y_{j}, x_{j+1}\right)$ for some $j$, or in $\left[-\infty, x_{1}\right)$ or in $\left(y_{m}, \infty\right]$. Assume $z$ is in $\left(y_{j}, x_{j+1}\right)$ for some $j$. Then $z \in\left(c_{j}, c_{j+1}\right)$ and therefore $h(z)=i_{j}$. Since $a^{f} \in B$, we have $a_{j}^{f} \leq y_{j}$ and $a_{j+1}^{f} \geq x_{j+1}$. Hence, $z$ is in $\left(a_{j}^{f}, a_{j+1}^{f}\right]$. Therefore, $f(z)=i_{j}$.

Assume now $z \in\left[-\infty, x_{1}\right)$. Since $c_{1} \geq x_{1}$ we have $z \in\left[-\infty, c_{1}\right]$. Therefore, $h(z)=i_{0}$. Since $a^{f} \in B$ we have $a_{1}^{f} \in\left[x_{1}, y_{1}\right]$. By transitivity, $z \in\left[-\infty, a_{1}^{f}\right]$. Therefore, $f(z)=i_{0}$. The case $z \in\left(y_{m}, \infty\right]$ is similar.

We are finally ready to characterize countably compact LOTS whose $C_{\mathrm{p}}(\cdot, n)$ are linearly Lindelöf for all $n$.

THeOREM 8. Let $L$ be a countably compact LOTS. Then $C_{\mathrm{p}}(L, n)$ is linearly Lindelöf for each $n$ iff $T(L)$ is linearly Lindelöf.

Proof. Necessity is given by Theorem 3. Let us prove sufficiency. Every continuous function from a countably compact LOTS to $n$ is constant or a step function with finitely many steps. Therefore, by Theorem 7 , it suffices to show that $(T(L))^{n}$ is linearly Lindelöf for each $n$. We proved earlier that $T(L)$ is Lindelöf (see discussion after Question 5). Let us show that $T(L)$ is a P-space. Let $S=\bigcap_{n} U_{n}$, where $U_{n}$ is open in $T(L)$ for each $n$. Fix $x \in S$. If $x \in L$ then $\{x\}$ is open, hence $x$ is in the interior of $S$. Suppose $x \in d L$. Assume $x \in(-\infty, \infty)$. Since base neighborhoods at $x$ in $T(L)$ are from the subspace topology on $T$, for each $n$ we can fix an interval $\left(a_{n}, b_{n}\right) \ni x$ such that $\left(a_{n}, b_{n}\right)_{T} \subset U_{n}$. We may assume that $a_{n}, b_{n} \in L$ and the $(n+1)$ st interval is inside the $n$th interval. Then $x \in \bigcap_{n}\left(a_{n}, b_{n}\right)_{T} \subset S$. If $x$ is not in the interior of $S$ then either $a_{n} \rightarrow x$ or $b_{n} \rightarrow x$. Since $x \notin L$, we arrived at a contradiction with countable compactness of $L$. Therefore, $x$ is in the interior of $S$.

Since $T(L)$ is a Lindelöf P-space, every finite power of $T(L)$ is Lindelöf. Hence $C_{\mathrm{p}}(L, n)$ is linearly Lindelöf for each $n$. 
Observe that if the Dedekind completion of $L$ is connected (= " $L$ has no gaps") then $T(L)=d L$.

Corollary 9. Let $L$ be a countably compact LOTS with connected Dedekind completion. Then $C_{\mathrm{p}}(L, n)$ is linearly Lindelöf for each $n$ iff $d L$ is linearly Lindelöf.

Let us demonstrate Theorem 8 in application. In [BUZ], the author proved that $C_{\mathrm{p}}(X)$ is Lindelöf if $X$ is a first-countable countably compact subspace of some ordinal $\tau$. Using Theorem 8 , it is easy to derive a weaker version of that result as follows. First, it is possible to introduce an order on $X$ that induces the topology of $X$. The space $T(X)$ is linearly Lindelöf because any uncountable subset of $X$ accumulates to some missing ordinal of uncountable cofinality in $\tau+1$. By Theorem $8, C_{\mathrm{p}}(X, n)$ is linearly Lindelöf for every $n$. Since our conclusion is weaker than the one in [BUZ] we do not give the details.

Twice in this paper we applied our results to derive only weaker versions of older results. This motivates the following questions.

Question 10. Let $X$ be a countably compact space and let $C_{\mathrm{p}}(X)$ be linearly Lindelöf. Is $C_{\mathrm{p}}(X)$ Lindelöf?

In general, it is interesting to know for which classes of spaces, linear Lindelöf and Lindelöf properties of $C_{\mathrm{p}}$ are equivalent.

QUESTION 11. Let $X$ be a first-countable countably compact space (or compact). Suppose $C_{\mathrm{p}}(X, 2)$ is Lindelöf. Is $C_{\mathrm{p}}\left(X, 2^{\omega}\right)$ Lindelöf? What if $C_{\mathrm{p}}(X, n)$ is Lindelöf for each $n$ ?

If we do not require first-countability then an answer to this question is consistently "no" even for a compact space. In [POL], R. Pol constructed an example of a separable zero-dimensional compactum $X$ such that $C_{\mathrm{p}}\left(X, 2^{n}\right)$ is Lindelöf for all $n$ while $C_{\mathrm{p}}\left(X, 2^{\omega}\right)$ is not. For first-countable pseudocompact spaces, the answer to Question 11 is consistently "no" too. In $[D \& S]$, A. Dow and P. Simon proved that under certain set-theoretic assumptions there exists a mad family $\mathcal{A}$ on $\omega$ such that the corresponding Mrówka space $\Psi(\mathcal{A})$ has $C_{\mathrm{p}}(\Psi(\mathcal{A}), 2)$ Lindelöf. Recall that any $\Psi$-space is first-countable and pseudocompact. In the same paper, the authors discovered that $C_{\mathrm{p}}\left(\Psi(\mathcal{A}), 2^{\omega}\right)$ is not Lindelöf for any mad family $\mathcal{A}$. They state their result as follows: " $C_{\mathrm{p}}(\Psi(\mathcal{A}))$ is not Lindelöf for any mad family $\mathcal{A}$ ". However, their proof needs from the reals only the existence of a non-trivial convergent sequence. Therefore, they actually proved much more, namely, that "the space $C_{\mathrm{p}}(\Psi(\mathcal{A})$, convergent sequence) is not Lindelöf for any mad family $\mathcal{A}$ ". 


\section{References}

[ARH] A. V. Arkhangel'skiı̌, Topological Function Spaces, Math. Appl. 78, Kluwer, Dordrecht, 1992.

[BUZ] R. Z. Buzyakova, In search for Lindelöf $C_{\mathrm{p}}$ 's, Comment. Math. Univ. Carolin. 45 (2004), 145-151.

[D\&S] A. Dow and P. Simon, Spaces of continuous functions over a $\Psi$-space, Topology Appl. 153 (2006), 2260-2271.

[ENG] R. Engelking, General Topology, rev. ed., Sigma Ser. Pure Math. 6, Heldermann, Berlin, 1989.

[MIS] A. S. Mishchenko, Finally compact spaces, Soviet Math. Dokl. 145 (1962), 11991202.

[NAH] L. B. Nahmanson, Lindelöfness in function spaces, in: Fifth Teraspol Symposium on Topology and its Applications, Kishinev, 1985, 183 (in Russian).

[POL] R. Pol, Concerning function spaces on separable compact spaces, Bull. Acad. Polon. Sci. Sér. Sci. Math. Astronom. Phys. 25 (1977), 993-995.

Mathematics Department

UNCG

PO Box 26170

Greensboro, NC 27402, U.S.A.

E-mail: Raushan_Buzyakova@yahoo.com

Received 18 August 2005;

in revised form 20 May 2006 